\title{
LIFTING COMMUTING 3-ISOMETRIC TUPLES
}

\author{
BENJAMIN RUSSO
}

Abstract. An operator $T$ is called a 3-isometry if there exists operators $B_{1}\left(T^{*}, T\right)$ and $B_{2}\left(T^{*}, T\right)$ such that

$$
Q(n)=T^{* n} T^{n}=1+n B_{1}\left(T^{*}, T\right)+n^{2} B_{2}\left(T^{*}, T\right)
$$

for all natural numbers $n$. An operator $J$ is a Jordan operator of order 2 if $J=U+N$ where $U$ is unitary, $N$ is nilpotent order 2 , and $U$ and $N$ commute. An easy computation shows that $J$ is a 3 -isometry and that the restriction of $J$ to an invariant subspace is also a 3 -isometry. Those 3 -isometries which are the restriction of a Jordan operator to an invariant subspace can be identified, using the theory of completely positive maps, in terms of a positivity condition on the operator pencil $Q(s)$. In this article, we establish the analogous result in the multi-variable setting and show, by modifying an example of Choi, that an additional hypothesis is necessary. Lastly we discuss the joint spectrum of sub-Jordan tuples and derive results for 3-symmetric operators as a corollary.

Mathematics subject classification (2010): Primary: 47A20; Secondary: 47A45, 47B99, $34 \mathrm{~B} 24$.

Keywords and phrases: Tuples, dilation theory, 3 -symmetric operators, 3 -isometric operators, nonnormal spectral theory, Taylor spectrum, complete positivity, Wiener-Hopf factorization, multi-variable.

\section{REFERENCES}

[1] Jim Agler, Subjordan operators, ProQuest LLC, Ann Arbor, MI, 1980. Thesis (Ph. D.) - Indiana University.

[2] Jim Agler and Mark Stankus, $m$-isometric transformations of Hilbert space, I, Integral Equations Operator Theory, 21 (4): 383-429, 1995.

[3] Joseph A. Ball and J. William Helton, Nonnormal dilations, disconjugacy and constrained spectral factorization, Integral Equations Operator Theory, 3 (2): 216-309, 1980.

[4] MAN Duen CHOI, Completely positive linear maps on complex matrices, Linear Algebra and Appl., 10: 285-290, 1975.

[5] Man-Duen Choi, Positive semidefinite biquadratic forms, Linear Algebra and its Applications, 12 (2): 95-100, 1975.

[6] Raúl E. CURTo, Applications of several complex variables to multiparameter spectral theory, in Surveys of some recent results in operator theory, Vol. II, volume 192 of Pitman Res. Notes Math. Ser, pages 25-90. Longman Sci. Tech., Harlow, 1988.

[7] J. William Helton, Jordan operators in infinite dimensions and Sturm Liouville conjugate point theory, Bull. Amer. Math. Soc., 78: 57-61, 1971.

[8] Scott McCullough and Benjamin Russo, The 3-isometric lifting theorem, Integral Equations and Operator Theory, pages 1-19, 2015.

[9] Vern PAUlsen, Completely bounded maps and operator algebras, volume $\mathbf{7 8}$ of Cambridge Studies in Advanced Mathematics, Cambridge University Press, Cambridge, 2002.

[10] VOLKER WROBEL, The boundary of Taylor's joint spectrum for two commuting Banach space operators, Studia Math., 84 (1): 105-111, 1986. 